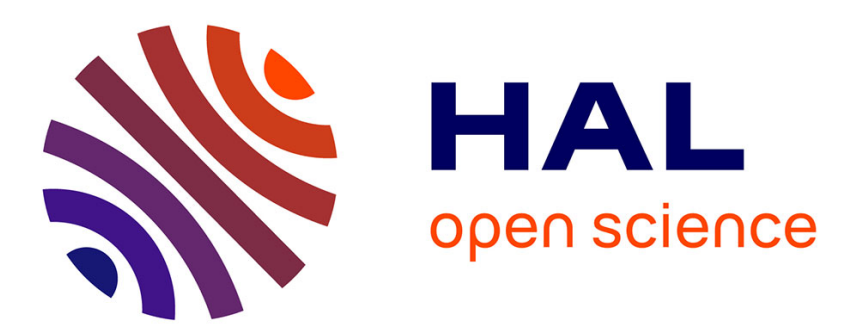

\title{
Alimentation hivernale des vaches allaitantes en zone de montagne
}

\author{
J.P. Garel, M. Petit, Jacques Agabriel
}

\section{To cite this version:}

J.P. Garel, M. Petit, Jacques Agabriel. Alimentation hivernale des vaches allaitantes en zone de montagne. Productions Animales, 1988, 1 (1), pp.19-23. hal-00895813

\section{HAL Id: hal-00895813 \\ https://hal.science/hal-00895813}

Submitted on 1 Jan 1988

HAL is a multi-disciplinary open access archive for the deposit and dissemination of scientific research documents, whether they are published or not. The documents may come from teaching and research institutions in France or abroad, or from public or private research centers.
L'archive ouverte pluridisciplinaire HAL, est destinée au dépôt et à la diffusion de documents scientifiques de niveau recherche, publiés ou non, émanant des établissements d'enseignement et de recherche français ou étrangers, des laboratoires publics ou privés. 
INRA Prod. Anim., $1988,1(1), 19-23$

\section{J.P. GAREL, M. PETTT*, J. AGABRIEL}

INRA Domaine de Marcenat

15190 Condat

* INRA Theix

Station des productions bovines

et chevalines

63122 Ceyrat

\section{Alimentation hivernale des vaches allaitantes en zone de montagne}

\section{Dans les élevages allaitants de montagne, peut-on réduire le niveau d'alimentation des vaches malgré la longueur de l'hiver?}

Dans les régions d'altitude (plus de $900 \mathrm{~m}$ ), les troupeaux de vaches allaitantes se sont développés principalement à partir des races rustiques : vaches Salers, Aubrac et croisées Charolais dans le Massif Central, vaches Gasconnes et croisées diverses dans les Pyrénées. Comme en plaine, les trois principaux objectifs techniques ayant une incidence directe sur le revenu tiré de ces troupeaux sont d'obtenir le maximum de veaux sevrés par vache et par an, de produire des veaux de qualités (poids, conformation, état sanitaire...) répondant aux besoins du marché, et de réduire au mieux les couts d'entretien du troupeau. Minimiser les couts est plus nécessaire encore que pour d'autres types d'animaux puisque la vache allaitante a un potentiel de production limité. Mais cela est plus difficile en altitude qu'en plaine. En particulier les conditions climatiques rigoureuses et la durée de l'hivernage élèvent les couts du logement et de l'alimentation hivernale.

\section{Résumé}

En zone de montagne plus encore qu'en plaine, il est nécessaire d'ajuster au mieux le niveau d'alimentation des vaches allaitantes pendant la période hivernale, afin d'économiser les stocks de fourrages récoltés. Avec les vaches adultes, une restriction des apports alimentaires est possible, au moins dans le cas des races rustiques. Mais elle doit rester modérée (de l'ordre de 1 UFL par jour) car la période de reproduction débute souvent au cours de l'hivernage en montagne, et la période de compensation au pâturage est courte. Cependant, les besoins alimentaires doivent être couverts pour les vaches en mauvais état à la rentrée à l'étable, ainsi que pour les vaches primipares.

\section{Caractéristiques de la conduite des vaches allaitantes en montagne}

Dans les régions de montagne, les vêlages ont lieu le plus souvent au cours du premier trimestre de l'année, un peu plus tôt qu'en plaine. Les deux grandes périodes, hivernage et paturage, qui rythment la vie annuelle du troupeau, sont de durées quasi-égales :

- Lhivernage dure de 5 mois et demi à 6 mois et demi selon les conditions locales, principalement selon l'altitude et l'exposition. Il couvre la fin de la gestation et le début de la lactation; la période de monte débute souvent avant la mise à l'herbe, et deux à trois semai- nes plus tôt encore pour les génisses que pour les vaches. La longueur de l'hivernage nécessite des stocks fourragers importants, alors même que la surface fauchée est moins productive qu'en plaine et fréquemment de dimension limitée. Il s'agit de foins dans la plupart des cas. Ils sont de qualité variable, mais souvent meilleure qu'en plaine (digestibilité, ingestibilité), principalement parce qu'ils sont récoltés à un stade moins tardif, ont séché un peu plus rapidement au sol et sont plus riches en azote. Mais, afin d'économiser les réserves fourragères, les vaches reçoivent des quantités de fourrages restreintes et sont le plus souvent volontairement sous-alimentées par rapport à leurs besoins réels. Pour couvrir leurs dépenses, elles mobilisent donc une partie de leur masse corporelle.

- La période de pâturage, de courte durée (moins de 7 mois), qui couvre l'essentiel de la période de lactation. Les vaches reconstituent alors leurs réserves corporelles tout en allaitant leur veau.

La sous-alimentation hivernale de la vache allaitante n'est envisageable que du fait de ses faibles besoins ; elle est en effet tarie pendant une grande partie de l'hivernage et sa production laitière est ensuite limitée, comprise par exemple entre 7 et $9 \mathrm{~kg}$ de lait par jour pour des vaches Salers et entre 6 et $8 \mathrm{~kg}$ pour des Aubrac. Il faut cependant remarquer que, par rapport aux vaches de races à viande de grand format, les vaches des races rustiques ont des besoins de production qui représentent une part plus importante de leurs besoins totaux, soit en raison d'un niveau de production laitière plus élevé (cas des Salers), soit en raison de leur format plus réduit (cas des Aubrac).

Nous tenterons de définir ici dans quelles limites et à quelles conditions il est possible de réduire les apports alimentaires hivernaux aux vaches allaitantes en montagne.

Des études sur la sous-alimentation hivernale des vaches allaitantes ont été menées au cours des dix dernières années sur les domaines INRA de Marcenat (Cantal) et de Laqueuille (Puy-de-Dôme) qui sont situés entre 1000 et $1200 \mathrm{~m}$ d'altitude. Beaucoup de ces études ont utilisé des vaches de race Salers. Selon les objectifs, elles étaient planifiées à l'échelle d'un hivernage, 


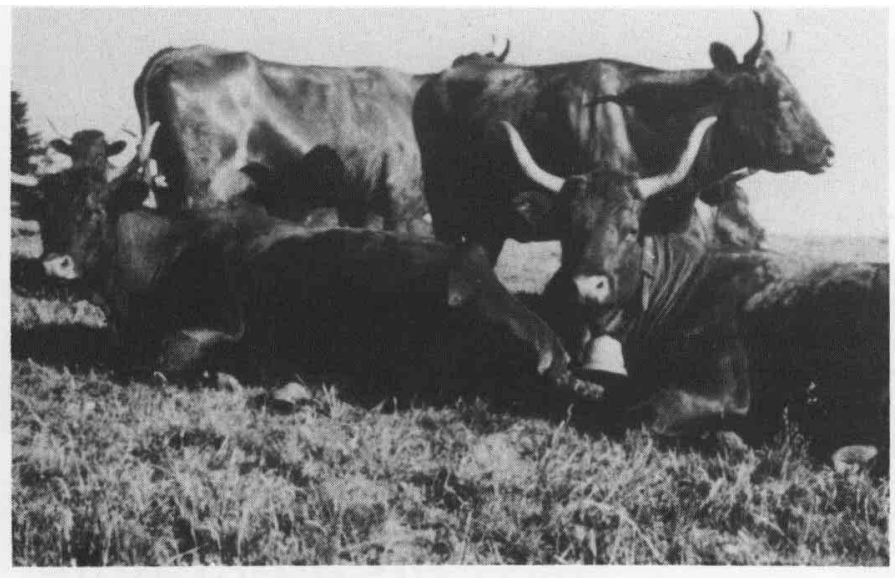

La vache allaitante sous-alimentée en hiver doit pouvoir reconstituer ses réserves corporelles au pâturage.

d'une année complète, ou de plusieurs années successives avec les mêmes vaches. Elles ont porté surtout sur la restriction des apports énergétiques.

\section{Une sous-alimentation raisonnée}

Lobjectif est d'économiser les fourrages hivernaux sans réduire la production du troupeau ni nuire à sa reproduction.

\section{En fin de gestation}

Les principales conséquences d'une sous-alimentation à la fin de la gestation sont maintenant bien connues. Un exemple en est donné dans le tableau 1 . Une sousalimentation relativement importante $(-1,5$ à -2 UFL/jour en moyenne au cours des 3 derniers mois) n'a pas d'incidence notable sur le poids et la vitalité du veau à la naissance, ce qui ne serait plus le cas avec une restriction alimentaire extrêmement sévère. La production laitière ultérieure de la vache n'est pas modifiée si celle-ci est alimentée normalement au-delà du vêlage. Il en est donc de même pour la croissance de son veau.

Cependant, un bas niveau alimentaire avant le vêlage (inférieur aux besoins d'environ 2 UFL par jour) risque de retarder les retours en chaleur si les vaches arrivent en mauvais état à la mise-bas : dans l'essai rapporté au tableau 1 , seulement $42 \%$ des vaches les plus sous-alimentées pendant la gestation ont des cycles ovariens 2 mois après le vêlage, au lieu de $65 \%$ de celles qui ont été sous-alimentées plus modérément. Le taux de gestation global à l'issue de la campagne de reproduction peut être réduit, et les vêlages suivants décalés.

\section{En lactation}

Si les réserves corporelles ne sont pas trop entamées avant le vêlage, une sous-alimentation modérée durant le début de la lactation ne diminue ni la production laitière des mères ni la croissance des veaux. Ce résultat s'explique à la fois par la faible quantité de lait que le veau est capable de boire dans son tout jeune age, et par la suite par l'effet stimulant de la tétée sur la sécrétion lactée. Ainsi, dans l'exemple du tableau 2, les vaches sous-alimentées de 1,5 à 2 UFL/jour ne diminuent pas significativement leur production de lait $(7,9 \mathrm{~kg}$ par jour au lieu de 8,7$)$ au cours des trois premiers mois de la lactation.

Il faut toutefois tempérer ce résultat selon l'importance et la durée de la sous-alimentation et selon le stade de lactation : avec des vaches qui ont vêlé en automne ou au tout début de l'hiver, une restriction alimentaire de 1,5 à 2 UFL/jour pendant les 3 à 4 derniers mois de l'hivernage réduit la persistance de la production laitière ; celle-ci peut juste avant la mise à l'herbe, être inférieure de $1 \mathrm{~kg}$ à celle de vaches alimentées selon leurs besoins.

Dans le cas des vaches vellant tôt en hiver, une sousalimentation trop importante après vêlage peut surtout compromettre leur aptitude à se reproduire avant la mise à l'herbe : dans l'exemple rapporté au tableau 2 , la réduction du niveau alimentaire a entraîné un accroissement important de l'intervalle entre vêlages, ains qu'une baisse sensible de la fertilité à l'œstrus induit.

C'est pourquoi avec des vaches en état moyen au vêlage, une sous-alimentation en lactation de $15 \%$ par rapport aux besoins ( -1 à $-1,5$ UFL par jour) est tolérable lorsqu'elles vêlent moins d'un mois et demi à deux mois avant la mise à l'herbe, mais ne l'est plus lorsqu'elles vêlent tôt, afin de ne pas décaler les vêlages suivants.

\section{Importance de l'état des vaches à la rentrée à l'étable}

La sous-alimentation hivernale n'est possible que dans la mesure où les vaches sont en état satisfaisant au début de l'hivernage. Elles disposent alors de réserves corporelles mobilisables. Ces réserves jouent le rỏle de

Tableau 1. Effets d'une sous-alimentation au cours des 3 derniers mois de la gestation (vaches Salers).

\begin{tabular}{|l|c|c|}
\hline Niveau de sous-alimentation & Modéré & Fort \\
\hline Apports énergétiques : & & 4,5 \\
- en UFL & 86 & 72 \\
- en \% des besoins & 607 & 597 \\
Poids vif initial (kg) & 29 & 16 \\
Gain de poids en gestation (kg) & -6 & -16 \\
Variation de masse corporelle (1) (kg) & 38 & 37 \\
Poids des veaux à la naissance (kg) & 65 & 42 \\
Vaches cyclées 60 jours après vêlage (\%) & 95 & 89 \\
\hline Taux de gestation global (\%) & & \\
\hline
\end{tabular}

d'après Petit 1978

(1) masse corporelle = poids vif diminué du poids du fœtus, des enveloppes et liquides foetaux, ainsi que du poids du contenu digestif. 
Tableau 2. Effets d'une sous-alimentation au cours des 3 premiers mois de la lactation (vaches Salers).

\begin{tabular}{|l|c|c|}
\hline Niveau de sous-alimentation & Nul & Modéré \\
\hline Apports énergétiques : & & 7,0 \\
- en UFL & 9,2 & 84 \\
- en \% des besoins & 105 & -52 \\
Variation de masse corporelle (1) (kg) & -25 & 7,9 \\
Production de lait (kg/j) & 8,7 & 63 \\
Vaches cyclées 60 jours après vêlage (\%) & 64 & 21 \\
Vêlages sur œestrus induit (\%) & 59 & 406 \\
Intervalle entre vêlages (jours) & 384 & \\
\hline
\end{tabular}

d'après Petit et Garel 1981

(1) masse corporelle $=$ poids vif - contenu digestif

tampon entre les dépenses de la vache et les apports alimentaires (tableaux 1 et 2). Elles sont en majorité lipidiques et sont reconstituées pendant la période de pâturage, notamment au printemps lorsque l'herbe est abondante et de bonne qualité.

La manifestation externe du phénomène de mobilisation/reconstitution des réserves corporelles est le changement d' "état" de l'animal. L'importance du tissu gras sous-cutané est un bon reflet de l'état des réserves lipidiques du corps entier. C'est pourquoi on utilise, dans la pratique, une note d' "état" établie après maniements à différents endroits précis (flancs, attache de la queue) de l'animal.

Nous avons comparé des vaches rentrées à l'étable en bon état (note 3/5) ou en mauvais état (note 1,5/5), qui ont été nourries durant tout l'hivernage selon deux niveaux d'alimentation énergétiques, de part et d'autre des besoins théoriques (tableau 3) : le poids des veaux à la naissance et la production laitière sont là encore peu dépendants de l'état nutritionnel. En revanche, la variation de la masse corporelle dépend non seulement des apports alimentaires hivernaux mais aussi de l'état initial des vaches : l'efficacité alimentaire globale est la meilleure dans le cas des vaches "maigres".

Les performances de reproduction des vaches dépendent également dans une large mesure de leur état initial : le retour en chaleur après vêlage des vaches rentrées "maigres" est significativement retardé, même quand elles sont correctement alimentées pendant l'hivernage et le taux de gestation des vaches maigres et sous-alimentées est insuffisant (70\% seulement) (tableau 3).

Les effets de la sous-alimentation dépendent donc non seulement de son intensité et de sa durée mais aussi de l'état initial des réserves corporelles de la vache.
Dans la pratique, on admet que pour une vache rentrée en état moyen à bon, la perte de poids vif entre la rentrée à l'étable et la fin de l'hivernage ne doit pas dépasser 8 à $9 \%$ du poids initial, soit environ $50 \mathrm{~kg}$ pour une vache Salers pesant $600 \mathrm{~kg}$ à l'automne. Cette perte de poids vif correspond à la fois au poids des produits de la conception à la rentrée à l'étable et à celui des réserves corporelles mobilisées au cours de l'hivernage.

Les vaches rentrées en mauvais état (note inférieure à 2/5) ne doivent pas perdre de poids vif. Elles seront pour cela nourries selon leurs besoins, et même audessus ( $+0,5$ à +1 UFL par jour) si elles vêlent tôt en hiver. On mesure là tout l'intérêt de rentrer les vaches à l'étable en état satisfaisant (note : 3/5), et donc de mettre auparavant à leur disposition un pâturage de qualité suffisante.

\section{Effets de niveaux d'alimentation répétés en hiver et au pâturage}

Une sous-alimentation modérée ( $-1,5$ UFL/jour) répétée au cours des hivers successifs n'affecte pas ou peu les performances des vaches allaitantes, à condition qu'elles puissent bénéficier au pâturage d'herbe abondante et de bonne qualité. C'est ainsi que dans un essai réalisé durant 4 lactations successives sur les mêmes vaches Salers, une réduction de 1,5 UFL par jour ( $20 \%$ en moyenne pour l'ensemble de l'hivernage) a eu peu d'effet sur la croissance moyenne des veaux de la naissance au sevrage bien qu'ils soient nés tôt en hiver, car la production laitière de leurs mères n'est réduite qu'en fin d'hivernage.

Limiter la quantité d'herbe pâturée, par exemple en augmentant le chargement de $30 \%$ au-dessus de la

Tableau 3. Effets combinés de l'état des vaches à la rentrée à l'étable et du niveau alimentaire hivernal (vaches Salers et Charolaises).

\begin{tabular}{|l|cc|cc|}
\hline Etat à l'entrée à l'étable (note sur 5) & \multicolumn{2}{|c|}{$\mathbf{3 , 0}$} & \multicolumn{2}{|c|}{$\mathbf{1 , 5}$} \\
\hline Niveau alimentaire hivernal & Haut & Bas & Haut & Bas \\
Variation de masse corporelle (1) $(\mathrm{kg})$ & -6 & -31 & +20 & -15 \\
Production laitière journalière en période hivernale (kg) & 9,6 & 9,5 & 9,4 & 9,2 \\
Vaches cyclées 50 jours après vêlages (\%) & 63 & 42 & 27 & 38 \\
Taux de gestation global (\%) & 85 & 91 & 91 & 70 \\
\hline
\end{tabular}

(1) masse corporelle $=$ poids vif diminué du poids des fotus, des enveloppes et liquides foetaux, ainsi que du poids du contenu digestif.

Rentrer les vaches en bon état permet de réduire les apports alimentaires hivernaux. 
Tableau 4. Effets pendant 4 années successives du niveau d'alimentation hivernale et du chargement durant la seconde moitié du pâturage (vaches Salers).

\begin{tabular}{|c|c|c|c|c|}
\hline \multirow{2}{*}{$\begin{array}{l}\text { Niveau d'alimentation hivernale } \\
\text { Niveau de chargement du pâturage } \\
\text { en automne }\end{array}$} & \multicolumn{2}{|c|}{$\begin{array}{c}\text { Haut } \\
\text { (besoins) }\end{array}$} & \multicolumn{2}{|c|}{$\begin{array}{c}\text { Bas } \\
\text { (besoins }-1,5 \text { UFL) }\end{array}$} \\
\hline & Normal & Fort & Normal & Fort \\
\hline Date moyenne de vêlage & $27 / 1$ & $29 / 1$ & $1 / 2$ & $3 / 2$ \\
\hline $\begin{array}{l}\text { Poids au sevrage des veaux (kg) } \\
\text { (moyenne mâles / femelles) }\end{array}$ & 312 & 302 & 304 & 284 \\
\hline Décalage des vêlages en 4 ans (1) & $\begin{array}{c}\text { Dernier vêlage } \\
\text { avancé } \\
\text { de } 7 \text { jours }\end{array}$ & $\begin{array}{c}\text { Dernier vêlage } \\
\text { retardé } \\
\text { de } 7 \text { jours }\end{array}$ & $\begin{array}{c}\text { Dernier vêlage } \\
\text { retardé } \\
\text { de } 7 \text { jours }\end{array}$ & $\begin{array}{c}\text { Dernier vêlage } \\
\text { retardé } \\
\text { de } 7 \text { jours }\end{array}$ \\
\hline $\begin{array}{l}\text { Gain de poids cumulé des vaches } \\
\text { ( } 4 \text { années) (kg) }\end{array}$ & 78 & 55 & 52 & 50 \\
\hline
\end{tabular}

Petit et Garel, non publié (1) par vache présente durant 4 lactations successives.

normale durant la seconde moitié de la saison de pâturage, a des effets globaux similaires à ceux d'une réduction du niveau d'alimentation hivernal (tableau 4 : comparaison des lot Haut-chargement fort et lot Baschargement normal). Dans ce cas, c'est surtout la diminution de la croissance automnale des veaux qui limite leur poids au sevrage. Mais si les apports alimentaires sont restreints à la fois au cours de l'hivernage et du pâturage, les performances du troupeau sont plus réduites encore car les vaches ont moins de réserves corporelles à la rentrée à l'étable : en particulier, le poids au sevrage des veaux est plus faible (tableau 4 : lot Baschargement fort), inférieur de 18 à $28 \mathrm{~kg}$ à celui des veaux issus des vaches mieux alimentées. Cette différence de poids au sevrage provient du décalage des naissances, de la réduction des croissances en hiver et au printemps (de $50 \mathrm{~g} / \mathrm{j}$ environ), et pendant la période de chargement élevé en automne (de 150 à $200 \mathrm{~g} / \mathrm{j}$ ).

Bien entendu, le handicap lié au chargement élevé du pâturage pourrait être partiellement compensé à court terme par un apport d'aliment concentré au veau, et, à plus long terme, par un tarissement plus précoce de sa mère ou par une meilleure alimentation hivernale. Il est cependant préférable dans le cas d'une alimentation restreinte au cours de plusieurs hivers successifs, que la vache allaitante puisse bénéficier de sai- sons de pâturage favorables pour reconstituer ses réserves corporelles, et atteindre un poids normal à l'âge adulte.

En définitive, les apports recommandés sont résumés au tableau 5 pour une vache de $600 \mathrm{~kg}$, rentrée à l'étable en état satisfaisant à bon $(2,5$ à 3$)$ ou en mauvais état (2 ou moins), et vêlant tôt en hiver ou plus tardivement. Ils admettent une sous-alimentation énergétique modérée ( -1 à $-1,5 \mathrm{UFL} / \mathrm{j}$ environ, soit 10 à $15 \%$ en-dessous des besoins) pour les vaches rentrées à l'étable en état satisfaisant et qui ne vêlent pas tôt en hiver. Cependant, les équilibres azoté, minéral et vitaminique doivent toujours être respectés. Les fourrages de montagne assurent généralement des apports azotés suffisants. Des déficits peuvent cependant apparaître avec des fourrages récoltés tardivement, notamment dans les zones d'altitude plus modeste. Dans ce cas, un apport azoté complémentaire (par exemple $0,5 \mathrm{~kg}$ de tourteau) est nécessaire pour assurer à la fois le bon fonctionnement de l'activité microbienne de la panse et pour satisfaire les besoins de l'animal.

\section{Cas particulier des vaches primipares}

Les jeunes vaches de trois ans rentrent le plus souvent à l'étable en état satisfaisant. Mais elles ont encore
Tableau 5. Apports quotidiens recommandés pour une vache allaitante adulte de $600 \mathrm{~kg}$ après vêlage.

\begin{tabular}{|c|c|c|c|c|}
\hline & \multicolumn{4}{|c|}{ Etat à la rentrée à l'étable } \\
\hline & \multicolumn{2}{|c|}{\begin{tabular}{l}
\multicolumn{2}{c}{ Moyen à bon } \\
UFL $\quad$ PDI
\end{tabular}} & \multicolumn{2}{|c|}{ Médiocre à mauvais } \\
\hline $\begin{array}{l}\text { Gestation (veau de } 40 \mathrm{~kg} \text { ) } \\
5^{\mathrm{e}} 6^{\mathrm{e}} \text { mois } \\
7^{\mathrm{e}} \text { mois } \\
8^{\mathrm{e}} \text { mois } \\
9^{\mathrm{e}} \text { mois }\end{array}$ & $\left.\begin{array}{l}4,7 \\
4,8 \\
5,4 \\
6,3\end{array}\right\} 5,6$ & $\left.\begin{array}{l}400 \\
410 \\
460 \\
535\end{array}\right\} 470$ & $\left.\begin{array}{l}5,0 \\
5,4 \\
6,1 \\
7,1\end{array}\right\} 6,3$ & $\left.\begin{array}{l}430 \\
460 \\
520 \\
595\end{array}\right\} 525$ \\
\hline $\begin{array}{l}\text { Lactation }\left(8 \mathrm{~kg} \text { de lait/jour au } 2^{\mathrm{e}} \mathrm{n}\right. \\
\text { - Vêlage précoce : } 1^{\mathrm{er}} \text { mois } \\
\qquad 2^{\mathrm{e}} \text { mois } \\
3^{\mathrm{e}}-4^{\mathrm{e}} \text { mois }\end{array}$ & $\begin{array}{l}7,3 \\
8,1 \\
8,8\end{array}$ & $\begin{array}{l}685 \\
760 \\
825\end{array}$ & $\begin{array}{c}8,6 \\
9,4 \\
10,0\end{array}$ & $\begin{array}{l}810 \\
880 \\
930\end{array}$ \\
\hline - Vêlage tardif : $\begin{array}{ll}1^{\mathrm{er}} \text { mois } & 2^{\mathrm{e}} \text { mois }\end{array}$ & $\begin{array}{l}6,7 \\
7,1\end{array}$ & $\begin{array}{l}630 \\
665\end{array}$ & $\begin{array}{l}8,2 \\
8,6\end{array}$ & $\begin{array}{l}770 \\
810\end{array}$ \\
\hline
\end{tabular}

d'après Petit 1988, adapté aux races rustiques et aux régions de montagne.

(1) 0,45 UFL et $53 \mathrm{~g}$ de PDI par $\mathrm{kg}$ de lait en plus ou en moins.

Note : Ces apports proposés doivent permettre d'obtenir une reproduction régulière (à intervalle d'un an) si les vaches ne sont pas maintenues entravées durant tout l'hiver mais peuvent sortir quasi-quotidiennement dès le début de la période de monte. 


\begin{tabular}{|l|c|c|}
\hline \multicolumn{1}{|c|}{ Apports énergétiques } & $\begin{array}{c}\text { Haut } \\
\text { (=besoins) }\end{array}$ & $\begin{array}{c}\text { Bas } \\
\text { (=besoins-1,5 UFL) }\end{array}$ \\
\hline Variation de poids hivernal (kg) & +4 & -43 \\
Gain de poids annuel des vaches de 30 à 42 mois (kg) & +16 & -5 \\
$\begin{array}{l}\text { Poids au sevrage des veaux (kg) } \\
\text { (moyenne mâles et femelles) }\end{array}$ & 277 & 259 \\
Intervalle vêlage-saillie fécondante (j) & 82 & 102 \\
\hline
\end{tabular}

Tableau 6. Effets d'une sous-alimentation hivernale de vaches Salers primipares.

Garel et Petit, non publié

des besoins de croissance importants. Celles de certaines races rustiques comme les Salers se caractérisent en outre par un niveau de production laitière relativement élevé ( 7 à $8 \mathrm{~kg} / \mathrm{j}$ au cours des 3 premiers mois de lactation). Une restriction des apports énergétiques hivernaux pour les vaches primipares, même modérée $(-1,5 \mathrm{UFL} / \mathrm{j})$, provoque une baisse de la croissance journalière des veaux de la naissance au sevrage, entraîne un retard des vêlages suivant pouvant atteindre 3 semaines, et un poids des vaches plus faible à 4 ans (tableau 6). Ce phénomène est souvent accentué par une durée d'allaitement plus longue que chez les vaches adultes, la date de vêlage étant généralement plus précoce chez les primipares.

De plus, la capacité d'ingestion des vaches primipares est plus faible que celle des vaches plus âgées (de 15 à $20 \%$ environ), si bien que lorsque les fourrages hivernaux sont de médiocre qualité, un complément d'aliment concentré est nécessaire pour éviter un décalage trop important du vêlage suivant. De même, l'appétit limité, la production laitière parfois importante (cas des Salers), et la courte saison de pâturage en altitude font que les vaches primipares sont peu capables de reconstituer à l'herbe - pendant la période d'allaitement - les réserves corporelles qu'elles ont perdu en stabulation. L'accumulation de ces difficultés entraîne une réduction des performances de production en $2^{\mathrm{e}}$ lactation : le gain de poids des veaux est identique ou parfois même inférieur à celui des veaux de $1^{\text {re }}$ lactation, comme cela a été constaté à Marcenat pour le troupeau Salers.

\section{Discussion et conclusion}

En montagne, il est possible de sous-alimenter modérément les vaches allaitantes durant la période hivernale, malgré sa longue durée. Il faut cependant qu'elles aient la possibilité de reconstituer leurs réserves corporelles au cours de la période de pâturage suivante, ou, à défaut, à l'échelle de plusieurs années.

La période favorable à la reprise de poids et d'état des vaches est évidemment la première moitié de la saison de pâturage, lorsque l'herbe est abondante. Mais si la quantité (ou la qualité) de l'herbe disponible durant la seconde moitié de la saison est faible, non seulement la croissance des veaux est alors ralentie, mais encore l'état des réserves corporelles des vaches à la rentrée à l'étable est compromis. Il peut en résulter à plus long terme un décalage des vêlages ainsi qu'une réduction de la production laitière des mères et de la croissance des veaux dans le jeune âge. On mesure là le handicap de la montagne, où la durée de végétation active est généralement plus courte qu'en plaine. Allonger la période de tarissement en automne à l'herbe pourrait aussi permettre de remettre les vaches en bon état, surtout celles qui ont vêlé tôt en hiver et dont les veaux peuvent être sevrés plus tôt. Mais la période précédant la rentrée à l'étable est délicate : la quantité d'herbe disponible est faible le plus souvent, les conditions climatiques sont parfois défavorables, et la rentrée est souvent retardée pour économiser les fourrages.

La conduite hivernale des primipares est plus difficile que celle des vaches plus âgées. Un bon état à 30 mois ne paraît pas suffisant pour atténuer les effets de la sous-alimentation autour du premier vêlage. Il est donc nécessaire de bien alimenter les vaches en première lactation pour éviter de compromettre leur carrière ultérieure. Mais même alimentées à volonté, elles ne peuvent satisfaire leurs besoins qu'avec des fourrages de bonne qualité, en raison de leur capacité d'ingestion plus faible.

Avec les vaches de races rustiques comme avec celles d'autres races, la réduction des apports alimentaires affecte tout d'abord l'état corporel de la vache, ensuite sa reproduction. Puis, si la sous-alimentation est plus importante, elle peut réduire la croissance du veau allaité. Enfin, seulement si elle est très sévère, elle peut compromettre la vitalité du nouveau-né.

On ne sait cependant pas dans quelle mesure les vaches de races à viande spécialisées, placées dans les mêmes conditions de montagne, peuvent subir les mêmes réductions des apports alimentaires hivernaux que celles de races rustiques ; ou si, avec une durée de pâturage limitée, elles doivent être mieux alimentées pour conserver une production normale.

\section{Remerciements}

Nous remercions l'ITEB de nous avoir permis d'utiliser des extraits d'un article publié dans "Avenir pour les races rustiques dans le Massif Central" par l'ITEB et les organismes régionaux Auvergne, Languedoc-Roussillon, Midi-Pyrénées.

\section{Références bibliographiques}

AGABRIEL J., PETIT M., 1987. Peut-on sous-alimenter les vaches allaitantes en hiver. in D. Micol Ed., "Forum Fourrages Auvergne 86".

AGABRIEL J., PETIT M., 1987. Recommandations alimentaires pour les vaches allaitantes. Bull. Tech. C.R.Z.V. Theix, INRA, 70, 153-166.

PETIT M., 1988. Alimentation des vaches allaitantes. in R. Jarrige Ed., "Alimentation des bovins, ovins et caprins", INRA Publ. Paris.

PETIT M., GAREL J.P., 1981. The effect of underfeeding in early lactation on the performances of suckled cows. in Noble and MacLeod Ed., "Report on suckler cow workers meeting, Dunsany, Ireland, 1981". 